CERN-TH/2003-105

hep-ph/0305074

\title{
MINIMAL THEORETICAL UNCERTAINTIES IN INFLATIONARY PREDICTIONS
}

\author{
Daniel J. H. Chung, ${ }^{a, b 1}$ Alessio Notari, ${ }^{c 2}$ Antonio Riotto ${ }^{d 3}$ \\ ${ }^{a}$ Theory Division, CERN, CH-1211 Geneva 23, Switzerland \\ ${ }^{b}$ Department of Physics, University of Wisconsin, Madison, WI 53706, USA \\ ${ }^{c}$ Scuola Normale Superiore, Piazza dei Cavalieri 7, Pisa I-56126, Italy \\ ${ }^{d}$ INFN, sezione di Padova, via Marzolo 8, Padova I-35131, Italy
}

During inflation, primordial energy density fluctuations are created from approximate de Sitter vacuum quantum fluctuations redshifted out of the horizon after which they are frozen as perturbations in the background curvature. In this paper we demonstrate that there exists an intrinsic theoretical uncertainty in the inflationary predictions for the curvature perturbations due to the failure of the well known prescriptions to specify the vacuum uniquely. Specifically, we show that the two often used prescriptions for defining the initial vacuum state - the Bunch-Davies prescription and the adiabatic vacuum prescription (even if the adiabaticity order to which the vacuum is specified is infinity) - fail to specify the vacuum uniquely in generic inflationary spacetimes in which the total duration of inflation is finite. This conclusion holds despite the absence of any trans-Planckian effects or effective field theory cutoff related effects. We quantify the uncertainty which is applicable to slow roll inflationary scenarios as well as for general FRW spacetimes and find that the uncertainty is generically small. This uncertainty should be treated as a minimal uncertainty that underlies all curvature perturbation calculations. PACS: 98.80.Cq, 95.35.+d, 4.62.+v

\footnotetext{
${ }^{1}$ E-mail: Daniel.Chung@cern.ch

${ }^{2}$ E-mail: a.notari@sns.it

${ }^{3}$ E-mail: antonio.riotto@pd.infn.it
} 


\section{Introduction}

Recent measurements of the Cosmic Microwave Background (CMB) temperature fluctuations [1] give strong support for the picture that inflation gave rise to a flat spatial geometry and the scale invariant energy density fluctuations on superhorizon scales needed for large scale structure formation. As a generic prediction of inflation, the primordial scale invariant energy density fluctuations on superhorizon scales can be calculated perturbatively once the model of inflation is specified, including the prescription for the vacuum (i.e. Fock basis) of the inflaton field.

To see what one means by specifying a vacuum in the canonical formalism, consider the simple case of a quantized scalar field $\phi$ in an FRW-type universe with the metric of the form

$$
d s^{2}=a^{2}(\tau)\left(d \tau^{2}-d \vec{x}^{2}\right)
$$

(conformal time coordinates) where we will restrict ourselves to flat spatial sections for simplicity. Without any nongravitational interactions, the field in the Heisenberg representation has an expansion

$$
\phi(\mathbf{x}, \tau)=\int \frac{d^{3} k}{(2 \pi)^{3 / 2} a(\tau)}\left[a_{k} h_{k}(\tau) e^{i k \cdot \mathbf{x}}+a_{k}^{\dagger} h_{k}^{*}(\tau) e^{-i k \cdot \mathbf{x}}\right]
$$

where the $a_{k}$ is the annihilation operator which annihilates the vacuum and defines the Fock space. Because the creation and annihilation operators obey the commutator $\left[a_{k_{1}}, a_{k_{2}}^{\dagger}\right]=\delta^{(3)}\left(k_{1}-k_{2}\right)$, the $h_{k}$ 's obey a normalization condition $h_{k} h_{k}^{*}-h_{k}^{\prime} h_{k}^{*}=i$ to satisfy the canonical field commutators (henceforth, all primes or dots on functions of $\tau$ refer to derivatives with respect to $\tau$ as usual). Because the Heisenberg equation of motion for $\phi$ will force $h_{k}(\tau)$ to obey a second order ordinary differential equation, it has two independent solutions, and hence there needs to be a boundary condition or prescription to specify $h_{k}$ thereby defining the vacuum state. 
Following the procedure in Minkowski space is one way of defining the vacuum (i.e. specifying $h_{k}$ ): define $h_{k}$ to be the positive frequency eigenvector of the time translational Killing vector. Because cosmologically interesting spacetimes do not have timelike Killing vectors, there is no a priori unique definition in specifying the vacuum even without any non-gravitational interactions. Indeed, even with a timelike Killing vector, as in de Sitter space, there can be some ambiguity $[2,3,4,5]$. More generally, the simple reason for a lack of a good definition of vacuum state is just a problem of lacking asymptotically free states. The classic studies of quantum fields in curved spacetime (for good reviews, see for example $[6,7,8,9]$ and references therein) had focused primarily on systematically identifying the ambiguities and exploring various prescriptions that can remove them whenever possible.

Two widely used prescriptions for defining vacuum are what is usually called the Bunch-Davies prescription [10] and the adiabatic vacuum prescription of Parker and Fulling $[11,12,8]$. The main point of this paper is to show that both prescriptions, even when the adiabatic order of the vacuum construction is infinity (i.e. the best one can do), do not specify the vacuum uniquely in most situations. This is true as well in one of the most important case of generic inflationary spacetimes ${ }^{4}$ in which the total duration of inflation is finite, despite the absence of any trans-Planckian effects or effective field theory cutoff related effects $[13,14]$. (For arguments against the trans-Planckian and cutoff effects, see $[15,16,17,18]$.) The simple reason is that both methods rely on an asymptotic definition of vacuum, leaving an ambiguity inherent in any asymptotic expansion. Note that the ambiguity that we are focusing on is also independent of the effects due to transition into inflation explored by Ref. [19, 20, 21]. In addition to showing the existence of an ambiguity, we estimate the uncertainty and its implication

\footnotetext{
${ }^{4}$ Here, the "vacuum" of the inflationary era is referring to a no particle state of curvature perturbations.
} 
for inflationary prediction of density perturbations.

This work should complement the recent efforts [13, 14, 21, 22, 23] to uncover small quantum effects for the CMB measurements. After all, to see whether small effects can be measured, one must understand the inherent theoretical uncertainty in the calculations. Our uncertainty should be viewed as a minimal uncertainty that underlies all of these calculations, if one accepts either the adiabatic vacuum formalism or the Bunch-Davies vacuum formalism.

In most generic situations, the uncertainty is extremely small. Indeed, in view of our work, it should be clear that the vacuum used in the work of Ref. [14] does not constitute the best adiabatic vacuum possible in a realistic inflationary scenario without dS invariance. From the adiabatic vacuum formalism, it should be considered an "excited" state. Even from a Hamiltonian minimization point of view of Ref. [16], one reaches a similar conclusion. To keep our presentation short as possible and to emphasize its independence from trans-Planckian issues, we will not discuss this point further in this paper. However, the reader should note that the existence of a cutoff does not change any of the results in this paper for the adiabatic vacuum.

The order of presentation is as follows. We start off by reviewing the physical reasons for the ambiguities of a quantum vacuum in a cosmological spacetime. We then define and estimate the generic uncertainties of the "Bunch-Davies" and the adiabatic vacuum. The following section gives an example of how nonperturbative quantities can be calculated (despite the usual inherent uncertainties) in an adiabatic vacuum in a very special limiting situation. In Sec. 5, we compute what can be seen as a slightly more precise uncertainty in the adiabatic vacuum during slow roll inflationary spacetimes. Finally, we summarize and conclude. 


\section{Ambiguities of vacua}

From a traditional particle physicist's point of view, vacuum can be defined as the state of no real particles. As has been well studied since 1960's (see for example [6, 7, 8, 9] and references therein), this notion of vacuum is well known to be ambiguous: particles cannot always be unambiguously defined in the presence of a background field. For example, suppose one defines particles as the eigenstates of the momentum operator. These states as wavefunctions must then necessarily be spacetime translation eigenstates. However, if there is no spacetime translational symmetry of the background spacetime, there cannot be such an eigenstate.

Even if the spacetime were flat Minkowski space, if one were to define particles empirically with an idealized detector, whether or not the detector registers particles depends in general on the motion of the detector. If the detectors were restricted to geodesics of the background spacetime, only in Minkowski spacetime, would all the geodesic detectors agree to no particle detection [8].

Because of this property of the Minkowski space, one may then try to argue that it is best to abandon the notion of a vacuous curved spacetime and treat it as a collection of gravitons in a Minkowski background. In this case, by definition the curved spacetime is not vacuous, although the state with background gravitons may be considered to be vacuous of some other field, say the inflaton field $\phi$. Unfortunately, partly due to $\phi$ interactions with the background graviton fields, one is again forced back to having some

ambiguities in defining the vacuum for $\phi$. Hence, seen this way, we see the problem of the ambiguity of the vacuum, say with respect to the field $\phi$, is not special to curved spacetime geometry, but to any situation in which there is a background field that interacts with the field $\phi$. Just as in any other interacting quantum field theory, one may try to define free asymptotic states for $\phi$ and the graviton, and treat the interactions 
perturbatively. This would work if there is an asymptotically flat region of spacetime. Unfortunately, the key difference in the curved background situations of interest to inflationary cosmology is that there are no such asymptotically flat regions. ${ }^{5}$ Hence, the problem of vacuum prescription can be rephrased as trying to define a vacuum state in the absence of free asymptotic states.

Note that instead of relying on a particle definition of vacuum which is inherently nonlocal and observer dependent (the number of particles detected by any physical detector can be zero in one frame but not in another, even in Minkowski space), one can characterize the vacuum in terms of vacuum expectation value of the stress tensor, which has the advantage of being covariant (if it is zero in one frame, it is zero in all frames of reference). However, even here, the stress tensor vacuum expectation value is sensitive to the ambiguities in the boundary conditions and renormalization prescriptions of the correlation functions. These boundary condition sensitivities then reflect the ambiguities of the vacuum.

\section{The "Bunch-Davies" Vacuum and the Adiabatic Vacuum}

In this section, we would like to explain the two commonly used prescriptions that will be the focus of this paper.

\section{1 "Bunch-Davies" vacuum}

In the FRW cosmological context, the most well known and appealing prescription for identifying the vacuum is what is commonly called the Bunch-Davies prescription, which states that the positive frequency mode function $h_{k}$ (see Eq. (2)) should match asymp-

\footnotetext{
${ }^{5}$ There may be a way of obtaining a physically sensible answer by artificially turning on and off the gravitation, but we will not pursue this line of reasoning in this work.
} 
totically to the Minkowski space prescription in the limit that the physical momentum $k / a$ is much larger than the background geometry curvature scale $\left(H=\dot{a}(\tau) / a^{2}\right)$ :

$$
\lim _{k /(a H) \rightarrow \infty} h_{k} \sim \frac{1}{\sqrt{2 k}} e^{-i k \tau}
$$

In some cases, this uniquely specifies the vacuum.

However, when this prescription is carried out at a fixed cosmological time (i.e. $k \rightarrow \infty$ with $a(t)$ fixed), then the vacuum is not unique even in the simplest situations. Since inflation generally did not last an infinitely long time, the Bunch-Davies prescription cannot be applied in the asymptotic past limit, and the ambiguities associated with $k \rightarrow \infty$ are nonvanishing. This is independent of the existence of cutoffs or any transPlanckian physics.

For example, consider a massless scalar field minimally coupled to Einstein gravity in a patch of de Sitter space

$$
S=\int d^{4} x \frac{a^{2}}{2}\left(\dot{\phi}^{2}-(\nabla \phi)^{2}\right)
$$

where $a(\tau)=-1 /(\tau H)>0$ with $1 / H>0$ being the $\mathrm{dS}$ radius. The resulting mode equation is

$$
\ddot{h}_{k}(\tau)+w_{k}^{2}(\tau) h_{k}(\tau)=0
$$

where dots stand for derivatives with respect to the conformal time $\tau$ and

$$
w_{k}^{2}=k^{2}-\frac{\ddot{a}}{a}=k^{2}-\frac{2}{\tau^{2}} .
$$

The general positive frequency mode solution is

$$
h_{k}=A_{k} \frac{e^{-i k \tau}}{\sqrt{2 k}}\left(1+\frac{i H}{(k / a)}\right)+B_{k} \frac{e^{i k \tau}}{\sqrt{2 k}}\left(1-\frac{i H}{(k / a)}\right)
$$

where $A_{k}$ and $B_{k}$ satisfies

$$
\left|A_{k}\right|^{2}-\left|B_{k}\right|^{2}=1
$$


due to the normalization conditions given below Eq. (2). At any given time $\tau$, one can impose that the modes become Minkowskian as $|(k / a) / H| \rightarrow \infty$. Explicitly, this amounts to a functional matching of the form

$$
h_{k} \sqrt{2 k} e^{i k \tau} \rightarrow 1
$$

for as $|k \tau| \rightarrow \infty$ for all $\tau<0$. Now, note that since $\tau \rightarrow-\infty$ in Eq. (7) gives

$$
h_{k} \rightarrow A_{k} \frac{e^{-i k \tau}}{\sqrt{2 k}}+B_{k} \frac{e^{i k \tau}}{\sqrt{2 k}}
$$

for all $k>0$, this uniquely specifies $A_{k}=1$ and $B_{k}=0$ for all $k>0$.

However, consider the situation in which we make the restriction $|\tau|<\left|\tau_{0}\right|$ where $\tau_{0}$ is the time at the "beginning" of inflation. Now, the limit $|(k / a) / H| \rightarrow \infty$ can be taken only by taking $k \rightarrow \infty$ with $a>a\left(\tau_{0}\right)$. This means that we only know, for example, that

$$
B_{k} \rightarrow \mathcal{O}\left(1 / k^{n}\right)
$$

with $n>0$ as $k \rightarrow \infty .{ }^{6}$ Therefore, if we just impose $k \rightarrow \infty$ with a bounded $a(\tau)$, the Bunch-Davies prescription does not uniquely specify a vacuum. Applied to the case of Eq. (7), one must allow the ambiguity

$$
B_{k} \lesssim \mathcal{O}\left(\frac{H}{k / a\left(\tau_{0}\right)}\right) .
$$

A useful quantity to characterize the properties of the quantum perturbations of a massless scalar field during inflation is the power spectrum. For a generic quantity $g(\mathbf{x}, \tau)$, which can expanded in Fourier space as

$$
g(\mathbf{x}, \tau)=\int \frac{d^{3} k}{(2 \pi)^{3 / 2}} e^{i k x} g_{k}(\tau),
$$

the power spectrum can be defined as

$$
\left\langle 0\left|g_{k_{1}}^{*} g_{k_{2}}\right| 0\right\rangle \equiv \delta^{(3)}\left(k_{1}-k_{2}\right) \frac{2 \pi^{2}}{k^{3}} \mathcal{P}_{g}(k)
$$

\footnotetext{
${ }^{6}$ Note that $\exp (-i k \tau)$ does not have an asymptotic expansion in real $k$ as $k \rightarrow \infty$ because of an essential singularity.
} 
where $|0\rangle$ is the vacuum quantum state of the system. This definition leads to the usual relation

$$
\left\langle 0\left|g^{2}(\mathbf{x}, t)\right| 0\right\rangle=\int \frac{d k}{k} \mathcal{P}_{g}(k)
$$

If we compute the variance of the perturbations of the $\phi$ field

$$
\begin{aligned}
\left\langle 0\left|(\phi(\mathbf{x}, \tau))^{2}\right| 0\right\rangle & =\int \frac{d^{3} k}{(2 \pi)^{3}}\left|\phi_{k}\right|^{2} \\
& =\int \frac{d k}{k} \frac{k^{3}}{2 \pi^{2} a^{2}}\left|h_{k}\right|^{2} \\
& =\int \frac{d k}{k} \mathcal{P}_{\phi}(k),
\end{aligned}
$$

we may infer the power spectrum of the fluctuations of the scalar field $\phi$ to be

$$
\mathcal{P}_{\phi}(k) \equiv \frac{k^{3}}{2 \pi^{2}}\left|\phi_{k}\right|^{2}
$$

Therefore, for a massless scalar field in de Sitter space, we obtain on superhorizon scales the power spectrum

$$
\mathcal{P}_{\phi}(k)=\left(\frac{H}{2 \pi}\right)^{2}\left|A_{k}-B_{k}\right|^{2}\left(\frac{k}{a H}\right)^{n_{\phi}-1},
$$

with $n_{\phi}=1$. From Eq. (18) we infer then that any ambiguity in the parameter $B_{k}$ implies an ambiguity in the power spectrum $\mathcal{P}_{\phi}$ of the form

$$
\left|\frac{\delta \mathcal{P}_{\phi}(k)}{\mathcal{P}_{\phi}(k)}\right| \simeq 2\left|\operatorname{Re} B_{k}\right| \simeq \mathcal{O}\left(e^{-\left(N_{0}-N_{k}\right)}\right),
$$

where $N_{k}$ denotes the number of $e$-foldings before the end of inflation when a given wavelength $\lambda=a / k$ leaves the horizon during the de Sitter stage and $N_{0}$ denotes the number of e-foldings before the end of inflation when the spacetime can be considered a vacuum (which has an upper bound of total number of e-foldings for inflation). This power spectrum can be seen as an approximation to the curvature perturbation power spectrum, and the vacuum here can be seen as the vacuum with respect to curvature 
perturbations. Length scales of interest for the the CMB anisotropies give $N_{k}$ of order of 60 , and therefore one expects the theoretical ambiguity on the power spectrum to be sizeable if the total duration of the de Sitter stage corresponds to a number of $e$-foldings not far from 60 . Of course, in this case it would be difficult to assume that the spacetime is in a vacuum state (see for example $[19,20,21]$ ).

\subsection{Adiabatic vacuum}

In situations in which the vacuum is defined through the notion of particles, the natural fundamental operator is the number operator which counts the number of particles with momentum k. Parker in Ref. [11] postulated certain reasonable conditions that the number operator for a scalar field must satisfy in a FRW universe. The conditions were as follows:

1. $N_{k}$ be Hermitian due to the counting interpretation.

2. When the expansion is stopped at any time (i.e. $\dot{a} / a=0$ ), the operator becomes the usual Minkowski number operator.

3. The vacuum expectation value of the number operator varies slowly as possible with time as the expansion rate $\dot{a} / a$ becomes arbitrarily slow.

The first two conditions are obviously reasonable, and give rise to the definition of the number operator as

$$
N_{k}\left(\tau_{1}\right) \equiv a_{k}^{\left(\tau_{1}\right) \dagger} a_{k}^{\left(\tau_{1}\right)}
$$

where the superscripted $\tau_{1}$ refers to the time at which the boundary condition for vacuum is set (or equivalently, the boundary conditions for $h_{k}(\tau)$ ).

The third condition is the statement that the vacuum should be defined as to keep the number of particles as unchanging as possible. This third condition is what Parker 
has called the minimization postulate [11] and was later developed further by Parker and Fulling [12]. The formalism developed based on satisfying these conditions is called the adiabatic vacuum formalism $[12,8]$.

It is important to note that to define the number operator $N_{k}$, one must define what one means by a particle at any given time, $\tau_{1}$. On the other hand, to have defined a vacuum at an earlier time $\tau_{0}<\tau_{1}$ (recall that Heisenberg representation states such as the vacuum are time independent), one needed a definition of a particle at time $\tau_{0}$. Hence, the present formulation requires that one define particles at two different times. This should be contrasted with the formalism of computing the vacuum expectation value of the stress tensor, where the definition of particles (or equivalently the vacuum) needs to be defined only once, since the stress energy tensor is a local quantity which does not rely on the basis of Fourier expansion. However, since we will be taking the same prescription for particles at time $\tau_{0}$ and time $\tau_{1}$ in such a way that the total number of conditions that has to be specified is the same as in the case of computing the vacuum expectation value of the stress tensor, this inherently nonlocal definition of vacuum will probably also minimize the growth of the stress tensor to a large extent. Nonetheless, it is not obvious how the conclusions of our analyses would differ if stress tensor vacuum expectation values are used instead of $N_{k}$ in carrying out the minimization postulate. We will defer this question to a future work and focus on the particle based formalisms in this paper.

The adiabatic formalism specifies the value and the first time derivative of the mode function $h_{k}$ at a fixed time for any fixed momentum. The boundary condition data is specified by matching to an asymptotic expansion (adiabatic expansion) of the mode equation to all orders in the asymptotic parameter (even though the expansion does not converge in general, by the very nature of an asymptotic expansion). In de Sitter space, for example, the infinite adiabatic order vacuum is identical (up to the uncertainties 
inherent to the adiabatic vacuum) to the Bunch-Davies vacuum. In inflationary spacetimes in which the scale factor cannot be made arbitrarily small due to the finite duration of the inflationary phase or in which the momentum cannot be made arbitrarily large due to a cutoff, the adiabatic formalism naively has a chance to still specify a unique vacuum, unlike the Bunch Davies prescription. However, we will find that the adiabatic prescription suffers from an ambiguity problem as well.

The construction of the adiabatic vacuum is as follows. First, define the concept of an adiabatic order as the power of $1 / T$ that results for any term in an $1 / T \rightarrow 0$ asymptotic expansion after one makes the transformation $\tau \rightarrow \tau$ and $d / d \tau \rightarrow T^{-1} d / d \tau$ in the differential equation for the modes $h_{k} .{ }^{7}$. Then, any prototypical mode equation of the form

$$
\ddot{h}_{k}(\tau)+w_{k}^{2}(\tau) h_{k}(\tau)=0
$$

with $w_{k}^{2}=k^{2}+m^{2} a^{2}(\tau)+(6 \xi-1) \ddot{a} / a$ (where $\xi$ is a constant and $m$ is the mass of $\phi$ ) turns into

$$
\frac{1}{T^{2}} \ddot{\breve{h}}_{k}(\tau)+\widetilde{w}_{k}^{2} \widetilde{h}_{k}(\tau)=0
$$

where $\widetilde{w}_{k}^{2} \equiv k^{2}+m^{2} a^{2}(\tau)+(6 \xi-1) \ddot{a} /\left(T^{2} a\right)$ and a tilde has been added to $h_{k}$ to be a reminder that this function carries a fictitious parameter $T$ (later this will be set to 1 at which time the function will be denoted as $h_{k}$ ). Now, make a change in variables from $\widetilde{h}_{k}$ to $W_{k}$ by writing

$$
\widetilde{h}_{k}=\frac{1}{\sqrt{2 W_{k}}} e^{\left(-i \int^{\tau} W_{k}\left(\tau^{\prime}\right) d \tau^{\prime} T\right)}
$$

(where the $T$ in the exponent should be noted) and from Eq. (22), we obtain a new differential equation

$$
W_{k}^{2}=\widetilde{w}_{k}^{2}-\frac{1}{2 T^{2}}\left[\frac{\ddot{W}_{k}}{W_{k}}-\frac{3}{2}\left(\frac{\dot{W}_{k}}{W_{k}}\right)^{2}\right] .
$$

\footnotetext{
${ }^{7}$ Note that the point about only the derivative being transformed has been missed by a recent paper [23].
} 
We can then define a map

$$
A\left[W_{k}^{[n]}\right]=\sqrt{\widetilde{w}_{k}^{2}-\frac{1}{2 T^{2}}\left[\frac{\ddot{W}_{k}^{[n]}}{W_{k}^{[n]}}-\frac{3}{2}\left(\frac{\dot{W}_{k}^{[n]}}{W_{k}^{[n]}}\right)^{2}\right]}
$$

which is a map that raises the adiabatic order by two and also define

$$
W_{k}^{[n+2]}=A\left[W_{k}^{[n]}\right]
$$

where the superscript denotes the adiabatic order and $W_{k}^{[0]} \equiv \sqrt{k^{2}+m^{2} a^{2}}$. All of this construction lead to an approximate mode equation solution good to $A$ th adiabatic order in asymptotic expansion in $1 / T \rightarrow 0$ of

$$
h_{k}^{[A]}=\frac{1}{\sqrt{2 W_{k}^{[A]}}} e^{\left(-i \int^{\tau} W_{k}^{(A)}\left(\tau^{\prime}\right) d \tau^{\prime} T\right)} .
$$

This asymptotic expansion solution will serve as a template, just to set the boundary conditions for the mode function $\widetilde{h}_{k}$ which can in general be written as

$$
\widetilde{h}_{k}(\tau)=A_{k} f_{k}(\tau)+B_{k} f_{k}^{*}(\tau)
$$

where $f_{k}$ are exact basis functions satisfying Eq. (22). Specifically, we define the Ath adiabatic (order) vacuum at time $\tau_{0}$ by using the boundary condition

$$
\begin{gathered}
\tilde{h}_{k}^{\tau_{0}}\left(\tau_{0}\right)=h_{k}^{[A]}\left(\tau_{0}\right)+\mathcal{O}\left(1 / T^{(A+1)}\right), \\
\dot{h}_{k}^{\tau_{0}}\left(\tau_{0}\right)=\dot{h}_{k}^{[A]}\left(\tau_{0}\right)+\mathcal{O}\left(1 / T^{(A+1)}\right),
\end{gathered}
$$

where the left-hand side is the exact mode solution to the prototypical differential equation Eq. (22) and the boundary conditions are enforced to only order $1 / T^{(A+1)}$ as $T \rightarrow \infty .^{8}$ After the boundary conditions are set, one sets the vacuum mode of Eq. (2) as

$$
\left.h_{k}^{\tau_{0}}(\tau) \equiv \widetilde{h}_{k}^{\tau_{0}}(\tau)\right|_{T=1}
$$

\footnotetext{
${ }^{8}$ The correction is order $1 / T^{(A+1)}$ and not of order $1 / T^{(A+2)}$ because there is an integration in the exponent.
} 
which removes the fictitious parameter $T$.

To see that this construction satisfies the minimization postulate if we take the vacuum adiabatic order $A$ to $\infty$, let us compute the vacuum expectation value of the number operator corresponding to the number density per mode. This requires a Bogoliubov transformation from the vacuum mode solution with the boundary condition at $\tau=\tau_{0}$ into the one with the boundary condition at $\tau=\tau_{1}$ (any later time at which the particles are no longer being created). Defining the Bogoliubov transformation as

$$
h_{k}^{\tau_{1}}(\tau)=\alpha_{k} h_{k}^{\tau_{0}}(\tau)+\beta_{k} h_{k}^{* \tau_{0}}(\tau)
$$

the number density of particles per momentum $k$ is given by $n_{k}=\left|\beta_{k}\right|^{2}$. If the vacuum in the past is defined at $\tau=\tau_{0}$ with infinite adiabatic order boundary condition and the vacuum today is defined at $\tau=\tau_{1}$ with infinite adiabatic order boundary condition, carrying out the Bogoliubov transformation with the solution written in the form Eq. (23), one finds

$$
{ }_{\tau_{0}}\left\langle 0\left|N_{k}\left(\tau_{1}\right)\right| 0\right\rangle_{\tau_{0}} \propto\left|\beta_{k}\left(\tau_{1}, \tau_{0}\right)\right|^{2}=\frac{1}{4 W_{k}^{\tau_{0}} W_{k}^{\tau_{1}}}\left\{\frac{1}{4}\left(\frac{\dot{W}_{k}^{\tau_{0}}}{W_{k}^{\tau_{0}}}-\frac{\dot{W}_{k}^{\tau_{1}}}{W_{k}^{\tau_{1}}}\right)^{2}+\left(W_{k}^{\tau_{0}}-W_{k}^{\tau_{1}}\right)^{2}\right\}(32
$$

where the right hand side (composed of exact solutions to Eq. (24)) can be evaluated at any $\tau$ and the superscripts indicate the time at which the boundary conditions were placed.

Now, the minimization postulate is satisfied if $\frac{d^{n}}{d t^{n}}\left({ }_{\tau_{0}}\left\langle 0\left|N_{k}\left(\tau_{1}\right)\right| 0\right\rangle_{\tau_{0}}\right)$ is minimized for all non-negative integers $n$ if the expansion rate can be turned off arbitrarily slowly. For any $a(\tau)$, we can affect the slowly turning off of the expansion rate by reintroducing the adiabatic order parameter $1 / T \rightarrow 0$ as before. Since by construction, the $W_{k}^{\tau_{i}}$ functions in Eq. (32) when expanded in $1 / T$ match the asymptotic expansions in $1 / T$ of $W_{k}^{[\infty]}$ of Eq. (26), as long as the asymptotic expansion is uniform in $\tau$ between $\tau_{0}$ and $\tau_{1}$ (no singularities in the asymptotic expansion occur between $\tau_{0}$ and $\tau_{1}$ ), the right hand side 
of Eq. (32) vanishes identically when expanded in $1 / T$. Hence $\frac{d^{n}}{d t^{n}}\left({ }_{\tau_{0}}\left\langle 0\left|N_{k}\left(\tau_{1}\right)\right| 0\right\rangle_{\tau_{0}}\right)$ will fall off faster than any finite power of $1 / T$ as $T \rightarrow \infty$, thus satisfying the minimization condition.

In general, one must remember that $W^{[\infty]}$ does not exist because the $W^{[A]}$ construction procedure generates an asymptotic expansion about a nonanalytic point rather than a convergent series. In other words, for a fixed order $A$ (and with $T=1$ ), there exists only an $A$-dependent region in time for which $W^{[A]}$ approximates well the exact solution to Eq. (5), with the leading error on the approximation growing with $A$ for any fixed time $\tau$. Hence, when the limit $A \rightarrow \infty$ is taken first, the time region in which the approximation is valid can shrink to 0 for a fixed $T=1$.

As an example of an adiabatic vacuum, let us apply this formalism to the case of massless scalar field in dS space (considered in Eq. (4)). The iteration map Eq. (25) produces up to sixth order

$$
\begin{aligned}
& W_{k}^{[0] 2}=k^{2} \\
& W_{k}^{[2] 2}=k^{2}\left[1-\frac{2}{(T k \tau)^{2}}\right] \\
& W_{k}^{[4] 2}=k^{2}\left[1-\frac{2}{(T k \tau)^{2}}+\frac{3}{(T k \tau)^{4}}+\mathcal{O}\left(\frac{1}{(T k \tau)^{6}}\right)\right] \\
& W_{k}^{[6] 2}=k^{2}\left[1-\frac{2}{(T k \tau)^{2}}+\frac{3}{(T k \tau)^{4}}-\frac{4}{(T k \tau)^{6}}+\mathcal{O}\left(\frac{1}{(T k \tau)^{8}}\right)\right] .
\end{aligned}
$$

The template asymptotic expansion $\widetilde{h}_{k}^{[A]}$ obtained from $W^{[A]}$ approximates the exact solution with an error of order $1 / T^{A+1}$. For example, one can write down explicitly using $W_{k}^{[4]}$ (dropping the higher order corrections to it)

$$
\tilde{h}_{k}^{[4]}=\frac{e^{-i k \tau T}}{\sqrt{2 k}}\left[1-\frac{i}{k \tau T}+\frac{2 i}{5 k^{5} \tau^{5} T^{5}}+\mathcal{O}\left(1 / T^{6}\right)\right]
$$


where the $1 / T^{5}$ term is the leading uncertain term (displayed just for clarity), and up to $1 / T^{4}$, the template function matches the first term of the exact solution

$$
\widetilde{h}_{k}=A_{k} \frac{e^{-i k \tau T}}{\sqrt{2 k}}\left(1-\frac{i}{(k \tau T)}\right)+B_{k} \frac{e^{i k \tau T}}{\sqrt{2 k}}\left(1+\frac{i}{(k \tau T)}\right) .
$$

Carrying out the matching procedure of Eq. (29) except up to 5th adiabatic order instead of the just the required 4 th, one finds

$$
\begin{aligned}
& A_{k}=1+\frac{3 i}{2 k^{5} \tau_{0}^{5} T^{5}}+\ldots \\
& B_{k}=0+\mathcal{O}\left(1 / T^{6}\right)
\end{aligned}
$$

where we have displayed one of the uncertain terms explicitly for clarity. Note that the coefficients in general depend on $\tau_{0}$ (when the boundary condition was placed), but the sensitivity to it is to higher adiabatic order. In general, the coefficients $A_{k}$ and $B_{k}$ will be of the form

$$
\begin{aligned}
& A_{k}=1+\mathcal{O}\left(1 / T^{A+1}\right), \\
& B_{k}=0+\mathcal{O}\left(1 / T^{A+1}\right)
\end{aligned}
$$

for an $A$ th order adiabatic vacuum. In the limit that $A \rightarrow \infty$, the uncertainty drops off faster than any finite power of $1 / T$.

Recall that in the Bunch-Davies prescription, one could not remove the uncertainty in the choice of $\left\{A_{k}, B_{k}\right\}$ if we did not have information to let $a(\tau) \rightarrow 0$, say because of the finite period of inflation. Since in the adiabatic prescription, the boundary conditions may be set to infinite adiabatic order at a finite time, let us see whether at finite initial time $\tau_{0}$ it is possible to define an infinite adiabatic vacuum without any ambiguity. By inspection, we can cast $W^{[A] 2}$ in the form

$$
W^{[A] 2}=k^{2} \sum_{n=0}^{A / 2}(-1)^{n}(n+1)\left(\frac{1}{(T k \tau)^{2}}\right)^{n}+\mathcal{O}\left(\frac{1}{T^{A+2}}\right) .
$$


This means that in the limit $A \rightarrow \infty, W^{[\infty] 2}$ is expressed as

$$
W^{[\infty] 2}=k^{2} \sum_{n=0}^{\infty}(-1)^{n}(n+1)\left(\frac{1}{k \tau T}\right)^{2 n}
$$

which converges to a simple function ${ }^{9}$

$$
W^{[\infty] 2}=k^{2} \frac{(k \tau T)^{4}}{\left(1+(k \tau T)^{2}\right)^{2}} .
$$

Miraculously, the series converges in this simple situation! $!^{10}$ Indeed, inserting $W^{[\infty]}$ into (27), we obtain at any time $\tau$

$$
h_{k}^{[\infty]}=\frac{e^{-i k \tau T}}{\sqrt{2 k}}\left(1-\frac{i}{k \tau T}\right),
$$

which in view of Eq. (29) will set $A_{k}=1$ and $B_{k}=0$ in Eq. (35) (note that we have normalized the function properly with the arbitrary integration constant freedom).

This seems to indicate that the vacuum has been uniquely specified. Unfortunately, this is not true since if an infinite adiabatic order vacuum with $T \rightarrow \infty$ is chosen to be $\widetilde{h}_{k}(\tau)$, we can always choose another vacuum

$$
\underline{\tilde{h}}_{k}=\sqrt{1+\left|B_{k}\right|^{2}} \widetilde{h}_{k}(\tau)+B_{k} \widetilde{h}_{k}^{*}(\tau)
$$

if $B_{k} \widetilde{h}_{k}^{*}(\tau)$ falls off faster than any finite power of $1 / T$. The infinite order adiabatic vacuum boundary conditions do not distinguish $\widetilde{h}_{k}$ and $\underline{\underline{h}}_{k}$. Indeed, this can be seen directly in Eq. (29) because the "equation" is asymptotic, up to terms that vanish faster than $A$ th power of $1 / T$, where in the infinite adiabatic order case $A=\infty$ : i.e. we should have written instead of Eq. (42), the equation

$$
h_{k}^{[\infty]}=\frac{e^{-i k \tau T}}{\sqrt{2 k}}\left(1-\frac{i}{k \tau T}\right)+\mathcal{O}\left(1 / T^{\infty}\right)
$$

\footnotetext{
${ }^{9}$ Notice that in the limit $\tau=\tau_{0} \rightarrow-\infty$ one recovers $W^{[\infty] 2}=k^{2}$, i.e. the infinite adiabatic order vacuum reduces to the $\mathrm{BD}$ vacuum.

${ }^{10} \mathrm{We}$ will later give an example where there is no convergence.
} 
where $\mathcal{O}\left(1 / T^{\infty}\right)$ indicates the nonperturbative term possibly dropped in the matching to the template function. Since $T$ follows every factor of $k$, we can guess the uncertainty by assuming an exponential function for $B_{k}$ as

$$
B_{k} \approx \exp \left(-k T /\left(a\left(\tau_{0}\right) H\left(\tau_{0}\right)\right)\right)
$$

which should be compared to Eq. (12). (Note that this would also serve as a good estimate of uncertainties even outside of inflationary phase.) This leads to

$$
\left|\frac{\delta \mathcal{P}_{\phi}(k)}{\mathcal{P}_{\phi}(k)}\right| \simeq \mathcal{O}\left(\exp \left(-e^{N_{0}-N_{k}}\right)\right),
$$

where we have used the same notation as Eq. (19). Because of the double exponential, the uncertainty is very quickly negligible as $N_{0}$ becomes larger than $N_{k}$.

In the case in which the expansion in $1 / T$ stops converging, as in the quasi-dS case, we may be able to estimate the uncertainty in the adiabatic vacuum formalism a little less arbitrarily (compared to Eq. (45)) as follows. Indeed, if at some adiabatic order $n_{*}$ the expansion in $1 / T$ stops converging (with $T=1$, the higher order terms give a larger correction to the mode solution than the $n_{*}$ th order term), this signifies that the nonadiabaticity in the system enters at the $n_{*}$ th derivative. Therefore, as far as assigning an uncertainty to the adiabatic vacuum is concerned, instead of using Eq. (45), we may estimate the uncertainty in fixing the vacuum to simply be the $n_{*}$ term in the asymptotic expansion. Although the uncertainty obtained this way is not "nonperturbative", it is not clear what the physical advantage is in artificially "turning off" the expansion (using $1 / T$ ) beyond the $n_{*}$ derivative which is in some sense the limit of adiabaticity characteristic of the physical system. We will later use this approach to compute the uncertainty for the slow roll inflationary case.

There is an exception to the existence of an uncertainty in the adiabatic vacuum formalism, which is already suggested by the estimate in Eq. (45) in the case that $a\left(\tau_{0}\right)=$ 
0. ${ }^{11}$ Namely, if the boundary conditions are set at a time in which the nonadiabaticity is identically 0 , then there is no expansion in $1 / T$, and hence there is no ambiguity in the adiabatic vacuum. Indeed, the loss of time translational invariance which is operationally at the heart of the uncertainty of the vacuum disappears at the time when the nonadiabaticity is identically 0 . The test of the disappearance of the nonadiabaticity is that $W^{[A]}$ for any $A$ becomes identical, or more explicitly

$$
W^{[A]}\left(\tau_{0}\right)=W^{[0]}\left(\tau_{0}\right)
$$

for all $A$ where $\tau_{0}$ is the time at which the vacuum is defined. This time $\tau_{0}$ typically is $\pm \infty$, which means that an asymptotic expansion in $\tau$ must be taken to match the boundary conditions. Hence, the reason why the ambiguity disappears in this exceptional case can also be seen as due to the asymptotic expansion being in time $\tau$ instead of $1 / T$, since the coefficients in Eq. (28) are time independent although they can be be $T$ dependent.

This exceptional case can be viewed as a meeting point of the adiabatic vacuum formalism and the Bunch-Davies formalism. Its spirit is similar to the Bunch-Davies formalism in that the asymptotic expansion can be in time $\tau$ instead of a fictitious adiabatic parameter $T$. However instead of matching on to a Minkowski prescription, it matches on to a zeroth adiabatic order WKB prescription as in the adiabatic vacuum formalism. Essentially, the adiabatic formalism states that although not in general, sometimes a unique vacuum can be defined in regions of spacetime where the frequency is adiabatically a constant to an arbitrarily good degree just as in the Bunch-Davies prescription, except with the frequency given by the leading order WKB ansatz instead of a Minkowski prescription (Minkowski prescription means that with the scale factor frozen at a fixed value).

\footnotetext{
${ }^{11}$ Here, we are not claiming $a\left(\tau_{0}\right)=0$ generically defines an adiabatic point, but merely that Eq. (45) suggests there are special points in spacetime where the uncertainty vanishes.
} 
The reader should be aware that the adiabatic formalism is compatible with the Bunch-Davies formalism. For example, this formalism always gives the same vacuum as the Bunch-Davies formalism whenever the Bunch-Davies formalism can define a unique vacuum. Also, we would like to comment that although we would like to extend the "uniqueness" to the case in which WKB iteration Eq. (25) converges for $n=\infty$ (i.e. $W^{[\infty]}(\tau)$ is well defined with $T$ fixed at a finite value), it is not obvious to us whether there is any reason to consider such situations in any special manner since the convergence of WKB iteration is not identical to the absence of nonadiabaticity. It merely means that the asymptotic expansion in $1 / T$ is being taken about an analytic point in the function generating the asymptotic expansion.

\section{An Example of Calculable Nonperturbative Par- ticle Production}

In this section, we will discuss a model from Ref. [24] which illustrates the adiabatic formalism in situations where nonperturbative particle production can be unambiguously calculated. Owing to the supposed background metric, the vacuum construction will be unique, and hence exponentially suppressed particle production will be calculable.

Before moving on to the discussion, we would like to alert the reader here that this model is also reviewed on pg. 70 of Ref. [8]. However, their usage of adiabatic prescription can be misleading (it was to us, at least). As a consequence of their loose usage, they give the wrong impression that they can calculate reliably an exponentially suppressed particle production even without taking to $\pm \infty$ the time at which the vacuum is specified. For example, one sees that the matching in their equation (3.117) involves taking $\lambda \rightarrow \infty$, which by itself results in a possibly exponentially suppressed ambiguity in the vacuum that we discussed in Eq. (45). However, as long as the vacuum is specified at $\tau_{0}=-\infty$, 
we agree with their results modulo their inconsequential phase error in their equation (3.124). We present our reanalysis here such that the reader would not be confused about the discrepancy of how Ref. [8] seems to claim that nonperturbative calculations can be unambiguously done in the context of the adiabatic vacuum formalism even without taking $\tau_{0} \rightarrow-\infty$ while we claim that such calculations cannot be done, strictly speaking, without being able to take such a limit for the time at which the vacuum is specified.

To see how the nonperturbative Bogoliubov coefficient arises in an exactly solvable model of Ref. [24], consider the conformally coupled mode equation (i.e. Eq. (21) with $\xi=1 / 6)$ where the scale factor is given by

$$
a^{2}(\tau)=\alpha^{2}+\beta^{2} \tau^{2}
$$

leading to the effective frequency

$$
w_{k}^{2}=m \beta \lambda+m^{2} \beta^{2} \tau^{2}
$$

where

$$
\lambda \equiv \frac{m \alpha^{2}}{\beta}+\frac{k^{2}}{m \beta} .
$$

Since we would like to carry out the adiabatic procedure, we scale the derivatives w.r.t. $\tau$ by $1 / T$ :

$$
\left(\frac{1}{T^{2}} \partial_{\tau}^{2}+w_{k}^{2}\right) \widetilde{h}_{k}=0
$$

For differential equations of the form

$$
\frac{d^{2} u}{d x^{2}}+\left(x^{2}+\tilde{\lambda}\right) u=0
$$

the general solution is the parabolic cylinder function

$$
u=D_{-(1+i \widetilde{\lambda}) / 2}[ \pm(1+i) x]
$$


and its complex conjugate where for our case, one should identify

$$
\begin{aligned}
& x=\sqrt{m \beta T} \tau, \\
& \tilde{\lambda}=T \lambda,
\end{aligned}
$$

where one notes that as $T \rightarrow \infty$, the solution is effectively doubly scaled as $\sqrt{T} \tau$ and $T \lambda$. Note also that the parabolic cylinder function $D_{p}(z)$ is defined by its boundary condition

$$
D_{p}(z) \sim z^{p} e^{-z^{2} / 4}
$$

as $z \rightarrow \infty$. However, this asymptotic expansion is not the same expansion as the $T \rightarrow \infty$ expansion since there is an effective double scaling of $\tau$ and $\lambda$.

Let us first see what the adiabatic vacuum procedure gives for the particle production. We begin by writing explicitly the exact mode function (solution to Eq. (51)) as

$$
\widetilde{h}_{k}=A_{k} f+B_{k} f^{*}
$$

where

$$
f(\tau)=\frac{T^{1 / 4}}{(2 m \beta)^{1 / 4}} e^{-\pi \lambda T / 8} D_{-(1-i \lambda T) / 2}[(i-1) \sqrt{m \beta T} \tau]
$$

which as one sees has the curious double scaling of $\tau$ and $\lambda$ mentioned before. We need to obtain an asymptotic expansion of the parabolic cylinder function $D_{p}(z)$ as $T \rightarrow \infty$, which corresponds to an asymptotic expansion as the complex order $p$ goes to $i \infty$. Unfortunately, we do not know of any systematic way of doing this because asymptotic expansion for $D_{p}$ known in the literature corresponds to either the limit

$$
\sqrt{m \beta T}|\tau| \gg \lambda T / 2
$$

or

$$
\lambda T / 2 \gg m \beta T \tau^{2},
$$


both of which are not satisfactory for generic $\tau$. However, for $\tau \rightarrow \pm \infty$ we can satisfy Eq. (59), and for $\tau \rightarrow 0$ we can satisfy Eq. (60). In reproducing the result of Audretsch and Schaeffer, we will focus on the limit $\tau \rightarrow \pm \infty$ satisfying Eq. (59).

In the limit of $\tau \rightarrow-\infty$, the parabolic cylinder function has the asymptotic formula

$$
\begin{aligned}
D_{p}(z) & \sim z^{p} e^{-z^{2} / 4}\left[1-\sum_{n=1} \mathcal{O}\left(p^{2 n} / z^{2 n}\right)+\ldots\right] \\
& =2^{-1 / 4+i \lambda T / 4} e^{\pi \lambda T / 8+i \pi / 8}(m \beta T)^{\frac{-1}{4}(1-i \lambda T)} \\
& \times|\tau|^{-(1-i \lambda T) / 2} e^{\frac{i m \beta T \tau^{2}}{2}}\left[1-\sum_{n=1} \mathcal{O}\left(\frac{(\lambda T)^{2 n}}{(m \beta T)^{n} \tau^{2 n}}\right)+\ldots\right]
\end{aligned}
$$

where

$$
\begin{aligned}
& z=(i-1) \sqrt{m \beta T} \tau, \\
& p=-(1-i \lambda T) / 2,
\end{aligned}
$$

giving

$$
f \sim \frac{e^{\frac{i m \beta T \tau^{2}}{2}}}{\sqrt{2 m \beta|\tau|}} 2^{i \lambda T / 4} e^{i \pi / 8}(m \beta T)^{i \lambda T / 4}|\tau|^{i \lambda T / 2}
$$

where one has to be careful to write

$$
(i-1) \sqrt{m \beta T} \tau=-(i-1) \sqrt{m \beta T}|\tau|
$$

for $\tau<0 .{ }^{12}$

Now, we construct the template function. Let us start with the zeroth order expansion

$$
W_{k}^{[0]}(\tau)=\sqrt{m \beta \lambda+m^{2} \beta^{2} \tau^{2}}
$$

which gives

$$
h_{k}^{[0]}=\frac{\left[2\left(\sqrt{\beta m} \tau+\sqrt{\lambda+\beta m \tau^{2}}\right)\right]^{-i \lambda T / 2}}{\sqrt{2 \sqrt{m \beta \lambda+m^{2} \beta^{2} \tau^{2}}}} \exp \left[\frac{-i T}{2} \sqrt{\beta m\left(\lambda+\beta m \tau^{2}\right)} \tau\right] .
$$

\footnotetext{
${ }^{12}$ We see that in Eq. (62), we cannot take $T \rightarrow \infty$ after taking $\tau \rightarrow-\infty$ as the $T$ expansion is out of control in that limit (i.e. $T$ limit and $\tau$ limit do not commute unless the $T$ expansion can be summed up).
} 
In the limit $\tau \rightarrow-\infty$, we therefore have

$$
h_{k}^{[0]} \sim \frac{1}{\sqrt{2 m \beta|\tau|}} \exp \left[-i \frac{m \beta \tau|\tau| T}{2}\right](\sqrt{m \beta}|\tau|)^{i \lambda T / 2}(\lambda)^{-i \lambda T / 2} .
$$

We can consider higher orders:

$$
\begin{aligned}
W_{k}^{[2]}= & \sqrt{\beta m\left(\lambda+\beta m \tau^{2}\right)}+\frac{\left(3 \beta m \tau^{2}-2 \lambda\right) \sqrt{\beta m\left(\lambda+\beta m \tau^{2}\right)}}{8 T^{2}\left(\lambda+\beta m \tau^{2}\right)^{3}}, \\
W_{k}^{[4]}= & \sqrt{\beta m\left(\lambda+\beta m \tau^{2}\right)}+\frac{\left(3 \beta m \tau^{2}-2 \lambda\right) \sqrt{\beta m\left(\lambda+\beta m \tau^{2}\right)}}{8 T^{2}\left(\lambda+\beta m \tau^{2}\right)^{3}} \\
& -\frac{\left(76 \lambda^{2}-732 \beta \lambda m \tau^{2}+297 \beta^{2} m^{2} \tau^{4}\right) \sqrt{\beta m\left(\lambda+\beta m \tau^{2}\right)}}{128 T^{4}\left(\lambda+\beta m \tau^{2}\right)^{6}} .
\end{aligned}
$$

In the limit that $\tau \rightarrow-\infty$, these have the expansions

$$
\begin{aligned}
& W_{k}^{[2]} \sim\left(\beta m|\tau|+\frac{\lambda|\tau|}{2 \tau^{2}}-\frac{\lambda^{2}|\tau|}{8 \beta m \tau^{4}}+\ldots\right)+\frac{1}{T^{2}}\left(\frac{3|\tau|}{8 \beta m \tau^{4}}+\frac{19 \lambda|\tau|}{16 \beta^{2} m^{2} \tau^{6}}+\ldots\right), \\
& W_{k}^{[4]} \sim W_{k}^{[2]}+\frac{1}{T^{4}}\left(\frac{-297|\tau|}{128 \beta^{3} m^{3} \tau^{8}}+\frac{4731 \lambda|\tau|}{256 \beta^{4} m^{4} \tau^{10}}+\ldots\right)
\end{aligned}
$$

where we note that each power of $1 / T^{n}$ has an expansion in $\frac{\lambda}{\beta m \tau^{2}}$ while the leading correction coming from $1 / T^{n}$ terms is $|\tau| /\left([\beta m]^{n-1} \tau^{2 n}\right)$. This means that even for a fixed finite $T$, we can write

$$
\lim _{\tau_{0} \rightarrow-\infty} W^{[A]}\left(\tau_{0}\right) / W^{[0]}\left(\tau_{0}\right)=1
$$

and hence we can conclude that this corresponds to an exceptional situation in which infinite adiabaticity occurs at the time $\tau_{0}=-\infty$ when the vacuum is set. At any finite time $\tau_{0}$, we would have to use the matching in expansion of $1 / T$, which can lead to nonperturbative ambiguity, but here, all the terms in $1 / T$ are effectively identically 0 . For completeness, we integrate to display the template function exponent. We find

$$
\begin{aligned}
T \int^{\tau} d x W_{k}^{[4]}(x)= & {\left[\frac{1}{2} \beta T m|\tau| \tau+\frac{\lambda T|\tau|}{2 \tau} \ln |\tau|+\ldots\right] } \\
& +\frac{1}{T}\left[\frac{-|\tau|}{\tau} \frac{3}{16 \beta m \tau^{2}}+\ldots\right]+\mathcal{O}\left(T^{-3}\right)
\end{aligned}
$$


which means that when exponentiated, both the $1 / T$ and $1 / \tau$ expansions are both well in control.

Because of Eq. (75), not only do Eqs. (69) and (65) match up to $\tau$ independent phases (which can always be chosen appropriately in the indefinite integral Eq. (27)), but the specification is unique without any nonperturbative ambiguity. Furthermore, we have from Eq. (75) that $h_{k}^{[0]}(\tau \sim-\infty)=h_{k}^{[\infty]}(\tau \sim-\infty)$, implying that the matched vacuum $h_{k}$ is an infinite adiabatic order vacuum. We shall denote this vacuum as

$$
h_{k}^{\tau_{0}=-\infty}(\tau)=\frac{1}{(2 m \beta)^{1 / 4}} e^{-\pi \lambda / 8} D_{-(1-i \lambda) / 2}[(i-1) \sqrt{m \beta} \tau]
$$

where we have denoted the placement of the boundary condition at $\tau_{0}=-\infty$.

Since we can read off the template function at $\tau=\infty$ from Eqs. (68) and (69), we can write

$$
h_{k}^{\tau_{1}=\infty}(\tau)=h_{k}^{\tau_{0}=-\infty}(-\tau)^{*}
$$

specifying the vacuum at a future time. By examining the asymptotic expansions 9.246 of $[25]$, we can then write

$$
h_{k}^{\tau_{0}=-\infty}(\tau>0)=\left[\frac{-\sqrt{2 \pi} e^{i \pi / 4} e^{-\pi \lambda / 4}}{\Gamma\left[\frac{1}{2}(1-i \lambda)\right]}\right] h_{k}^{\tau_{1}=\infty}(\tau)+\left[-i e^{-\pi \lambda / 2}\right] h_{k}^{\tau_{1}=\infty *}(\tau),
$$

giving $^{13}$ a particle production coefficient of

$$
\beta_{k}=-i e^{-\pi \lambda / 2}
$$

More explicitly, if we had not set $T=1$ through Eq. (30) we could write $\beta_{k}=-i e^{-\pi T \lambda / 2}$, which shows explicitly that this coefficient is 0 to all orders in $1 / T$. Therefore, this nonperturbatively suppressed particle production could not have been reliably calculated if we had to make an expansion in $1 / T$ at any point in the calculation (we have made an expansion in $1 / T$ in this section just to show that it was not necessary as can be seen

\footnotetext{
${ }^{13}$ There is an error in the book by Birrell and Davies [8] for the first coefficient.
} 
for example in Eq. (75)). Such an expansion in $1 / T$ would have been necessary if the time $\tau_{0}$ at which vacuum is specified were finite.

\section{The adiabatic vacuum in the slow roll spacetime}

For any realistic scenario of inflation, the background spacetime is quasi-dS, unlike the exact dS that we treated explicitly before. By quasi-dS, we mean the Hubble rate $\left(H=\dot{a}(\tau) / a^{2}(\tau)\right)$ is not exactly constant, but changes with time as $\dot{H} / a=-\epsilon H^{2}$, where $\epsilon$ is one of the so-called slow-roll parameters. ${ }^{14}$ The other useful slow-roll parameter is $\eta=\left(V^{\prime \prime} / 3 H^{2}\right)$ where $V(\phi)$ is the inflaton potential and primes denote derivatives with respect to the inflaton field $\phi$. We wish to estimate the uncertainty in the vacuum in such a spacetime, and thereby estimate the uncertainty in the inflationary prediction of density perturbations.

In the gauge invariant treatment of cosmological perturbations generated during inflation, it is useful to define the gauge invariant Mukhanov variable $u=a \delta \phi^{(\mathrm{GI})}+z \Psi$ [26] where $z=\dot{\phi} / H$ and $\delta \phi^{(\mathrm{GI})} \Psi$ are the gauge invariant inflaton fluctuation and the Bardeen gravitational potential respectively [27]. Performing for the variable $u$ a field expansion similar to Eq. (1), one finds the mode equation (after inserting the adiabatic parameter $1 / T)$

$$
\frac{1}{T^{2}} \ddot{\tilde{h}}_{k}(\tau)+\left[k^{2}-\frac{(2+p)}{T^{2} \tau^{2}}\right] \tilde{h}_{k}(\tau)=0,
$$

where $p \equiv 3(3 \epsilon-\eta)$. The exact mode equation solution can be written in terms of Hankel functions as

$$
\tilde{h}_{k}=\sqrt{\frac{\pi}{2}} \sqrt{-\tau T}\left[A_{k} H_{\nu}^{(1)}(-k T \tau) e^{i \frac{\pi}{2}\left(\frac{1}{2}+\nu\right)}+B_{k} H_{\nu}^{(2)}(-k T \tau) e^{-i \frac{\pi}{2}\left(\frac{1}{2}+\nu\right)}\right]
$$

where one must be careful to note that $-\tau>0$ (we have also taken out $T k \tau$ independent

\footnotetext{
${ }^{14}$ Recall we are using conformal time coordinates.
} 
phases from $A_{k}$ and $B_{k}$ for convenience and normalized conveniently). Here, the order is defined as $\nu \equiv \sqrt{9+4 p} / 2 .{ }^{15}$

Let us now construct the adiabatic vacuum following the recipe of subsection 3.2. After rescaling $d / d \tau \rightarrow(1 / T) d / d \tau$ in the mode equation, the template frequencies can be constructed as

$$
\begin{aligned}
W_{k}^{[0] 2}(\tau) & =k^{2} \\
W_{k}^{[2] 2}(\tau) & =k^{2}\left[1-\frac{(2+p)}{(k \tau T)^{2}}\right] \\
W_{k}^{[4] 2}(\tau) & =k^{2}\left[1-(2+p) \frac{1}{(k \tau T)^{2}}+\left(3+\frac{3}{2} p\right) \frac{1}{(k \tau T)^{4}}+\mathcal{O}\left(\frac{1}{(k \tau T)^{6}}\right)\right], \\
W_{k}^{[6] 2}(\tau) & =k^{2}\left[1-(2+p) \frac{1}{(k \tau T)^{2}}+\left(3+\frac{3}{2} p\right) \frac{1}{(k \tau T)^{4}}+\left(-4+\frac{7}{2} p\right) \frac{1}{(k \tau T)^{6}}\right. \\
& \left.+\mathcal{O}\left(\frac{1}{(k \tau T)^{8}}\right)\right] .
\end{aligned}
$$

However, unlike in the dS case, the series is generally non-convergent for any finite $\tau=\tau_{0}$. As we discussed before, this type of nonconvergence is more generic than the convergent case.

To fourth order in adiabatic expansion, we find the following WKB template function

$$
h_{k}^{[4]}=\frac{e^{-i k \tau T}}{\sqrt{2 k}}\left(1+\frac{-i(1+p / 2)}{k T \tau}-\frac{p}{4 k^{2} T^{2} \tau^{2}}-\frac{i p}{6 k^{3} T^{3} \tau^{3}}+\frac{5 p}{24 k^{4} T^{4} \tau^{4}}\right) \text {. }
$$

For the asymptotic expansion of the Hankel functions $H_{\nu}^{(1,2)}$, we have from Ref. [25]

$$
\begin{aligned}
& H_{\nu}^{(1)}(z)=\sqrt{\frac{2}{\pi z}} e^{i\left(z-\frac{\pi}{2} \nu-\frac{\pi}{4}\right)}\left[\sum_{k=0}^{n-1} \frac{(-1)^{k}}{(2 i z)^{k}} \frac{\Gamma\left(\nu+k+\frac{1}{2}\right)}{k ! \Gamma\left(\nu-k+\frac{1}{2}\right)}+\theta_{1} \frac{(-1)^{n}}{(2 i z)^{n}} \frac{\Gamma\left(\nu+n+\frac{1}{2}\right)}{\Gamma\left(\nu-n+\frac{1}{2}\right)}\right], \\
& H_{\nu}^{(2)}(z)=\sqrt{\frac{2}{\pi z}} e^{-i\left(z-\frac{\pi}{2} \nu-\frac{\pi}{4}\right)}\left[\sum_{k=0}^{n-1} \frac{1}{(2 i z)^{k}} \frac{\Gamma\left(\nu+k+\frac{1}{2}\right)}{k ! \Gamma\left(\nu-k+\frac{1}{2}\right)}+\theta_{2} \frac{1}{(2 i z)^{n}} \frac{\Gamma\left(\nu+n+\frac{1}{2}\right)}{\Gamma\left(\nu-n+\frac{1}{2}\right)}\right],
\end{aligned}
$$

\footnotetext{
${ }^{15}$ The Hankel's functions are defined as $H_{\nu}^{(1)}=J_{\nu}+i Y_{\nu}$ and $H_{\nu}^{(2)}=H_{\nu}^{(1) *}$, where $J_{\nu}$ and $Y_{\nu}$ are, respectively, the Bessel functions of the first and the second kind.
} 
where $\operatorname{Re}(\nu)>-1 / 2,|\arg (z)|<\pi$ and $\theta_{1,2}$ are coefficients smaller than unity in front of the remainders. A useful formula is

$$
\frac{\Gamma\left(\nu+k+\frac{1}{2}\right)}{\Gamma\left(\nu-k+\frac{1}{2}\right)}=\frac{\left(4 \nu^{2}-1^{2}\right)\left(4 \nu^{2}-3^{2}\right) \ldots\left(4 \nu^{2}-(2 k-1)^{2}\right)}{2^{2 k}} .
$$

Expanding to first order in $p$ and fourth order in $T$, one can then compute

$$
\begin{aligned}
\tilde{h}_{k}(\tau) & \approx \frac{1}{\sqrt{2 k}}\left[\left(A_{k} f+B_{k} f^{*}\right)+\frac{i}{k \tau T}\left\{-A_{k}\left(1+\frac{p}{2}\right) f+B_{k}\left(1+\frac{p}{2}\right) f^{*}\right\}\right. \\
& -\frac{p}{4(k \tau T)^{2}}\left\{A_{k} f+B_{k} f^{*}\right\}+\frac{i p}{6(k \tau T)^{3}}\left\{-A_{k} f+B_{k} f^{*}\right\} \\
& \left.+\frac{5 p}{24(k \tau T)^{4}}\left\{A_{k} f+B_{k} f^{*}\right\}\right],
\end{aligned}
$$

where $f \equiv e^{-i k \tau T}$. Matching $\tilde{h}_{k}$ and $h_{k}^{[4]}$ just by inspection (comparing Eqs. (87) and (91)), one sees that $A_{k}=1$ and $B_{k}=0$ as expected.

Just to check further, suppose we took the 4 th order template $h_{k}^{[4]}$ and expanded to 6 th order in $T^{-1}$ and matched it to $\tilde{h}_{k}$ to 6 th order (instead of just to 4 th order). To accomplish this, note that since $W^{[4]}$ only needs to be expanded to $T^{-6}$ accuracy even though it is being multiplied by $T$ since the next order term in $W^{[4]}$ expansion is $T^{-8}$, resulting in a correction of $T^{-7}$ which we are ignoring anyway. We find

$$
h_{k(6 \mathrm{th})}^{[4]}=h_{k}^{[4]}+\frac{e^{-i k \tau T}}{\sqrt{2 k}}\left[\frac{-3 i}{8 k^{5} \tau^{5} T^{5}}(4+3 p)+\frac{9+5 p}{4 k^{6} \tau^{6} T^{6}}\right] .
$$

This results in

$$
\begin{aligned}
& A_{k}=1+\frac{3(2+p) i}{4 k^{5} T^{5} \tau_{0}^{5}}+\mathcal{O}\left(T^{-6}\right), \\
& B_{k}=\frac{-15(2+p)}{8 k^{6} T^{6} \tau_{0}^{6}} e^{-2 i k T \tau_{0}},
\end{aligned}
$$

where $\tau_{0}$ is the time at which the boundary conditions are placed. We see explicitly the order of the residual corrections to the 4 th order asymptotic expansion, and it is as expected. One can easily check similarly that the 6th adiabatic order WKB template 
function also results in $A_{k}=1$ and $B_{k}=0$ to 6 th order in $1 / T$. More explicitly, we have the template function

$$
h_{k}^{[6]}=h_{k}^{[4]}+\frac{e^{-i k \tau}}{\sqrt{2 k}}\left[\frac{3 i p}{8 k^{5} \tau^{5} T^{5}}-\frac{7 p}{8 k^{6} \tau^{6} T^{6}}\right]
$$

that fixes the vacuum. Indeed, the fact $A_{k}=1$ and $B_{k}=0$ is no surprise since the formulae Eqs. (88) and (89) and the template functions are the same asymptotic expansions to any given order.

The matching at time $\tau_{0}$ and at any given adiabatic order $A$ of the template function $h_{k}^{[A]}$ with the mode function (82) suffers of an ambiguity introduced by the nonvanishing remainders (proportional to $\theta_{i}$ ) displayed in expansions (88) and (89). As discussed earlier, the order at which the remainder stops converging (with $T=1$ ) can be attributed to be the extent to which the vacuum is nonadiabatic. Hence, instead of a guessed nonperturbative uncertainty of Eq. (45), we can set $T=1$ and compute the uncertainty that is due to the intrinsic nonadiabaticity of the background spacetime. (This is something we discussed at the end of Sec. 3.2.)

More explicitly, we can look for the adiabatic order $n_{*}$ at which the (absolute values of the) remainders are minimized, and then say that we cannot define the vacuum more certainly than this order due to the nonadiabaticity. Expanding the remainders to firstorder in the slow-roll parameters, we can express the absolute value of the remainder as

$$
R(n) \equiv\left|\frac{(-1)^{n} \Gamma(\nu+n+1 / 2)}{(2 i k|\tau|)^{n} \Gamma(\nu-n+1 / 2)}\right| \approx \frac{p \Gamma(2+n) \Gamma(n-1)}{3 \cdot 2^{n}\left(k\left|\tau_{0}\right|\right)^{n}}+\mathcal{O}\left(p^{2}\right) .
$$

We see clearly that the remainder vanishes in the dS limit of $p=0$, which corresponds to $\epsilon=\eta=0$. This is another way of understanding why in the pure de Sitter case we have been able to construct a convergent $W^{[\infty]}$. At a formal level, it merely means that the asymptotic expansion point $1 / T \rightarrow 0$ is an analytic point, allowing a convergent asymptotic expansion. 
We can find the minimum of $R$ at the location by taking a discrete derivative $R\left(n_{*}+\right.$ 1) $-R\left(n_{*}\right)=0$. Although $n_{*}$ will not lie at an integer value in general, the actual solution will be at the nearest integer. ${ }^{16}$ We thus find

$$
n_{*}=\operatorname{integer}\left(\frac{-1}{2}+\frac{1}{2} \sqrt{1+8\left(1+k\left|\tau_{0}\right|\right)}\right) \approx \operatorname{integer}\left(\sqrt{2 k\left|\tau_{0}\right|}\right)
$$

to be the order at which the uncertainty is minimized where the integer function finds the nearest integer value of the argument. Note that the value is independent of $p$ to leading order because in the limit that $p$ vanishes, the corrections identically vanish, giving an "arbitrary" $n_{*}$. In terms of e-folds, we can write

$$
n_{*} \simeq \sqrt{2} \exp \left(\left(N_{0}-N_{k}\right) / 2\right)
$$

where $N_{k}$ denotes the number of $e$-foldings from the time when a given wavelength $\lambda=a / k$ leaves the horizon $(\lambda=1 / H)$ till the end of inflation and $N_{0}$ denotes the number of $e$-foldings from the time the vacuum was set to the end of inflation. As we already mentioned, length scales of interest for the the CMB anisotropies give $N_{k}$ of order of 60 .

The corresponding ambiguity in the value of the parameter $B_{k}$ can be calculated from $R(n)$ as follows. We can consider the template function to be

$$
h_{k}^{\left[n_{*}-1\right]} \equiv \sqrt{\frac{\pi|\tau|}{2}} H_{\nu}^{(1)}(-k \tau) e^{i\left(\frac{1}{2}+\nu\right) \frac{\pi}{2}}+r_{n_{*}},
$$

where $r_{n}$ is related to the remainder as

$$
\begin{aligned}
r_{n} & =h_{k}^{[0]} \tilde{R}_{1}(n), \\
h_{k}^{[0]} & =\frac{e^{-i k \tau}}{\sqrt{2 k}}, \\
\tilde{R}_{1}(n) & =\frac{\sqrt{2}(-1)^{n} \Gamma\left(\nu+n+\frac{1}{2}\right) e^{-i\left(\frac{\pi}{2} \nu+\frac{\pi}{4}\right)}}{(2 i|\tau| k)^{n} \Gamma\left(\nu-n+\frac{1}{2}\right)},
\end{aligned}
$$

\footnotetext{
${ }^{16}$ When solving, however, one will find it convenient to assume self-consistently that $n_{*}$ will in the end be at an integer value.
} 
where $\left|\tilde{R}_{1}(n)\right|=\sqrt{2} R(n)$. Now, solving for the coefficients $A_{k}$ and $B_{k}$ using the system

$$
\begin{aligned}
h_{k}^{\left[n_{*}-1\right]}\left(\tau_{0}\right) & =\left.\tilde{h}_{k}\left(\tau_{0}\right)\right|_{T=1}, \\
h_{k}^{\left[n_{*}-1\right]^{\prime}}\left(\tau_{0}\right) & =\left.\tilde{h}_{k}^{\prime}\left(\tau_{0}\right)\right|_{T=1}
\end{aligned}
$$

gives

$$
B_{k} \approx-i h^{[0] 2} \frac{d \tilde{R}_{1}\left(n_{*}\right)}{d \tau}
$$

The ambiguity in the power spectrum $\mathcal{P}_{\mathcal{R}}$ of the comoving curvature perturbation $\mathcal{R}=$ $u / z$ is given by

$$
\left|\frac{\delta \mathcal{P}_{\mathcal{R}}(k)}{\mathcal{P}_{\mathcal{R}}(k)}\right| \simeq 2\left|\operatorname{Re} B_{k}\right| \simeq \mathcal{O}\left[p \exp \left(-2 \sqrt{2} e^{\left(N_{0}-N_{k}\right) / 2}-\left(N_{0}-N_{k}\right) / 2\right)\right],
$$

where we are using the notation and approximation explained below Eq. (19). Note that this is generically slightly larger than Eq. (46) and that it artificially vanishes in the limit that $p \rightarrow 0$. In the case that $p \rightarrow 0$, the best estimate that we can give for the uncertainty is Eq. (46), which is a merely a guessed function that drops off nonperturbatively fast. The theoretical ambiguity on the power spectrum of the comoving curvature perturbation is sizeable if the total duration of the de Sitter stage corresponds to a number of $e$-foldings not far from 60 . Of course, in this regime, it may not be a good approximation to treat the spacetime to be void of fluctuations.

\section{Conclusions}

Within the context of Bunch-Davies vacuum formalism and the adiabatic vacuum formalism we have answered the following question: "What is the minimal theoretical uncertainty in the inflationary curvature perturbation calculation if we assume that the curvature perturbation state at sometime $\tau_{0}$ near the beginning of inflation was a slow roll vacuum?" Even without any trans-Planckian effects, effective field theory cutoff 
related effects, or nongravitational field interaction effects, there is a minimal uncertainty in the curvature perturbation predictions of inflation coming from the inability to uniquely specify a vacuum (due to gravitational interactions). Within the adiabatic vacuum formalism, the power spectrum uncertainty is given by Eq. (106), and in more general situations (applicable to outside of the inflationary regime) by Eq. (45). The Bunch-Davies formalism gives a larger uncertainty of Eq. (19).

The minimum uncertainty presented here applies to all of the efforts $[13,14,21,22]$ to obtain measurable small effects on the CMB. In practice of comparing with data, there will certainly be other theoretical uncertainties, not only from other interaction effects of the inflaton, but reheating historical uncertainties [28] as well as astrophysical uncertainties which will most likely overwhelm the minimal uncertainty presented here, unless the number of $e$-foldings is very close to the minimum required for inflation (e.g. around 60). Even in that case, however, most likely, it will be difficult to assume that the curvature perturbation quantum state is that of a vacuum due to other energy density fluctuations present which depend on the history leading to the initiation of inflation. Nonetheless, it is important and reassuring to know that the inflationary vacuum in the conservative sense has very little ambiguity contrary to the impressions given particularly by Ref. [14]. Indeed, the main qualitative conclusion one can draw from our work is that even with a finite period of inflation, we can in most cases neglect the uncertainty associated with the vacuum when defined according to the most reasonable particle based definitions.

Finally, we would like to comment that the general idea that the vacuum is uncertain with a finite period of inflation is not entirely new (see for example [7, 29]). Here, we merely tried to quantify this in the context of slow roll inflationary models and show explicitly that the best estimates for the uncertainty lead us not to worry about this effect unless the total duration of inflation is close to 60 e-folds or so. 


\section{ACKNOWLEDGMENTS}

We would like to thank J. Cline, M. Einhorn, L. Everett, E. Kolb, F. Larsen, R. Leigh, L. Parker, R. Rattazzi, J. Van der Schaar, L. Senatore and I. Tkachev for conversations regarding this topic. We would like to thank in particular M. Einhorn and L. Parker for their comments on an early manuscript of this work.

\section{References}

[1] C. L. Bennett et al., arXiv:astro-ph/0302207.

[2] N. A. Chernikov and E. A. Tagirov, Annales Poincare Phys. Theor. A 9, 109 (1968).

[3] E. A. Tagirov, Annals Phys. 76, 561 (1973).

[4] E. Mottola, Phys. Rev. D 31, 754 (1985).

[5] B. Allen, Phys. Rev. D 32, 3136 (1985).

[6] B. S. Dewitt, Phys. Rept. 19, 295 (1975).

[7] S.A. Fulling, Aspects of Quantum Field Theory in Curved Spacetime (Cambridge University Press, Cambridge, England, 1989); Gen. Rel. Grav. 10 (1979) 807.

[8] N. D. Birrell and P. C. W. Davies, Quantum Fields in Curved Space (Cambridge University Press, Cambridge, 1982).

[9] R. M. Wald, Quantum Field Theory In Curved Space-Time And Black Hole Thermodynamics (Univ. Chicago Press, Chicago, 1994). 
[10] T. S. Bunch, J. Phys. A 13, 1297 (1980).

[11] L. Parker, Phys. Rev. 183, 1057 (1969).

[12] L. Parker and S. A. Fulling, Phys. Rev. D 9, 341 (1974).

[13] J. Martin and R. H. Brandenberger, Phys. Rev. D 63, 123501 (2001) [arXiv:hepth/0005209]; J. C. Niemeyer, Phys. Rev. D 63, 123502 (2001) [arXiv:astroph/0005533]; L. Mersini, M. Bastero-Gil and P. Kanti, Phys. Rev. D 64, 043508 (2001) [arXiv:hep-ph/0101210]; A. Kempf and J. C. Niemeyer, Phys. Rev. D 64, 103501 (2001) [arXiv:astro-ph/0103225]; R. Easther, B. R. Greene, W. H. Kinney and G. Shiu, Phys. Rev. D 64, 103502 (2001) [arXiv:hep-th/0104102]; R. Easther, B. R. Greene, W. H. Kinney and G. Shiu, Phys. Rev. D 67, 063508 (2003) [arXiv:hep-th/0110226]; R. H. Brandenberger and J. Martin, Int. J. Mod. Phys. A 17, 3663 (2002) [arXiv:hep-th/0202142]; S. Shankaranarayanan, Class. Quant. Grav. 20 (2003) 75 [arXiv:gr-qc/0203060]; S. F. Hassan and M. S. Sloth, arXiv:hepth/0204110; K. Goldstein and D. A. Lowe, Phys. Rev. D 67, 063502 (2003) [arXiv:hep-th/0208167].

[14] U. H. Danielsson, Phys. Rev. D 66, 023511 (2002) [arXiv:hep-th/0203198]; JHEP 0207, 040 (2002) [arXiv:hep-th/0205227]; L. Bergstrom and U. H. Danielsson, JHEP 0212, 038 (2002) [arXiv:hep-th/0211006]; R. Easther, B. R. Greene, W. H. Kinney and G. Shiu, Phys. Rev. D 66, 023518 (2002) [arXiv:hep-th/0204129].

[15] N. Kaloper, M. Kleban, A. E. Lawrence and S. Shenker, Phys. Rev. D 66, 123510 (2002) [arXiv:hep-th/0201158]; N. Kaloper, M. Kleban, A. Lawrence, S. Shenker and L. Susskind, JHEP 0211, 037 (2002) [arXiv:hep-th/0209231]; T. Banks and L. Mannelli, Phys. Rev. D 67, 065009 (2003) [arXiv:hep-th/0209113]; M. B. Einhorn and F. Larsen, Phys. Rev. D 67, 024001 (2003) [arXiv:hep-th/0209159]. 
[16] V. Bozza, M. Giovannini and G. Veneziano, arXiv:hep-th/0302184.

[17] A. A. Starobinsky, Pisma Zh. Eksp. Teor. Fiz. 73, 415 (2001) [JETP Lett. 73, 371 (2001)] [arXiv:astro-ph/0104043].

[18] A. A. Starobinsky and I. I. Tkachev, JETP Lett. 76, 235 (2002) [Pisma Zh. Eksp. Teor. Fiz. 76, 291 (2002)] [arXiv:astro-ph/0207572].

[19] L. P. Grishchuk and Ya. B. Zeldovich, Astron. Zh. 55, 209 (1978).

[20] M. S. Turner, Phys. Rev. D 44, 3737 (1991).

[21] C. P. Burgess, J. M. Cline, F. Lemieux and R. Holman, JHEP 0302, 048 (2003) [arXiv:hep-th/0210233].

[22] J. Maldacena, arXiv:astro-ph/0210603.

[23] C. Armendariz-Picon and E. A. Lim, arXiv:hep-th/0303103.

[24] J. Audretsch and G. Schäfer, Phys. Lett. 66A (1978) 459.

[25] I. S. Gradshteyn and I. M. Ryzhik, Tables of Integrals, Series, and Products (Academic Press, San Diego, 1980).

[26] See for instance, V. F. Mukhanov, H. A. Feldman and R. H. Brandenberger, Phys. Rept. 215, 203 (1992); D. H. Lyth and A. Riotto, Phys. Rept. 314, 1 (1999) [arXiv:hep-ph/9807278]; A. Riotto, arXiv:hep-ph/0210162.

[27] J. M. Bardeen, Phys. Rev. D 22 (1980) 1882.

[28] B. Feng, X. Gong and X. Wang, arXiv:astro-ph/0301111.

[29] A. D. Dolgov, M. B. Einhorn and V. I. Zakharov, Acta Phys. Polon. B 26, 65 (1995) [arXiv:gr-qc/9405026]. 